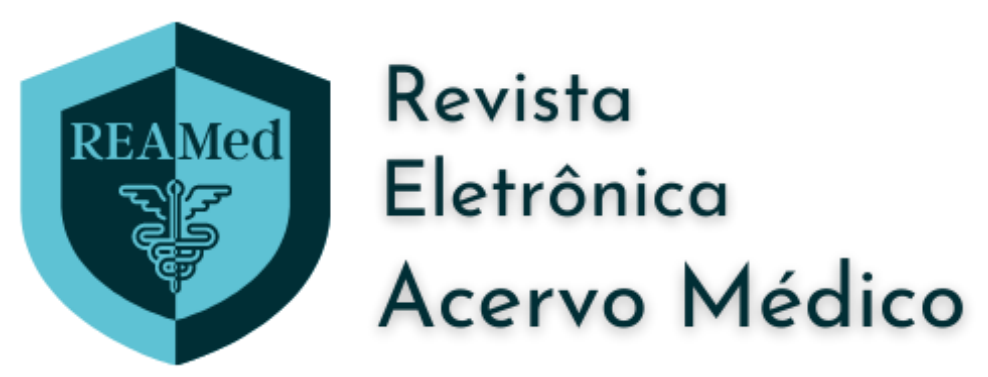

\title{
Prescrição de psicofármacos em pacientes pediátricos: uma revisão de literatura
}

\author{
Prescription of psychiatric drugs in pediatric patients: a literature review \\ Prescripción de fármacos psiquiátricos en pacientes pediátricos: una revisión de la literatura \\ Carla Resende Vaz Oliveira ${ }^{1 *}$, Caique Nunes Moreira ${ }^{1}$, Bruno Cezario Costa Reis ${ }^{1}$.
}

\section{RESUMO}

Objetivo: Analisar o padrão das prescrições dos psicofármacos em crianças, relacionando com a idade e gênero. Avaliando, assim, a existência ou não de prescrições inadequadas. Métodos: A abordagem metodológica deste trabalho se propõe a um compilado de pesquisa bibliográfica de abordagem qualitativa e caráter descritivo por meio de uma revisão integrativa da literatura nas bases de dados National Library of Medicine, Biblioteca Virtual em Saúde e Directory of Open Access Journals. Os descritores utilizados foram "over prescribing", "psychotropic drugs" e "child". Os critérios de inclusão foram artigos de journal article, clinical trial, ensaios clínicos, randomizados ou não randomizados, estudos de caso-controle, estudo de coorte, livre acesso, publicados em inglês, português, espanhol, no intervalo de 2010 a 2021 e faixa etária de 2 a 18 anos. Resultados: Os psicof armácos apresentados são os ansiolíticos, antipsicóticos, antidepressivos, antiepléticos e psicoestimulantes utilizados para controle dos comportamentos externalizantes e comportamentos internalizantes. Os externalizantes por sua vez são prevalentes em meninos mostrando divergência das prescrições e sintomatologia segundo as idades e sexo abordados. Considerações finais: Dessa forma, a prescrição de psicotrópicos é inadequada e indiscriminada na maioria dos casos, não respeitando as idades mínimas para prescrição, o diagnóstico e as indicação de cada medicamento e classe.

Palavras-chave: Prescrição inadequada, Psicofármacos, Infância.

\section{ABSTRACT}

Objective: To analyze the pattern of prescriptions for psychotropic drugs in children, relating to age and gender. Thus, evaluating the existence or not of inadequate prescriptions. Methods: The methodological approach of this work proposes a compilation of bibliographic research with a qualitative approach and descriptive character through an integrative literature review in the National Library of Medicine, Virtual Health Library and Directory of Open Access Journals databases. The descriptors used were "over prescribing", "psychotropic drugs" and "child". Inclusion criteria were journal article articles, clinical trial, clinical trials, randomized or non-randomized, case-control studies, cohort study, open access, published in English,

\footnotetext{
1 Universidade de Vassouras, Vassouras - RJ. *E-mail: carlarvaz4@gmail.com
} 
Portuguese, Spanish, between 2010 and 2021 and range aged from 2 to 18 years. Results: The psychopharmaceuticals presented are anxiolytics, antipsychotics, antidepressants, antieplectics and psychostimulants used to control externalizing and internalizing behaviors. Externalizing agents, in turn, are prevalent in boys, showing divergence of prescriptions and symptoms according to the ages and sex addressed. Final considerations: Thus, the prescription of psychotropic drugs is inadequate and indiscriminate in most cases, not respecting the minimum ages for prescription, diagnosis and indication of each drug and class.

Key words: Over prescribing, Psychotropic drugs, Childhood.

\section{RESUMEN}

Objetivo: Analizar el patrón de prescripción de psicotrópicos en niños, en relación con la edad y el sexo. De esta forma, se evalúa la existencia o no de prescripciones inadecuadas. Métodos: El enfoque metodológico de este trabajo propone una recopilación de investigación bibliográfica con enfoque cualitativo y carácter descriptivo a través de una revisión bibliográfica integradora en las bases de datos de la Biblioteca Nacional de Medicina, Biblioteca Virtual en Salud y Directorio de Revistas de Acceso Abierto. Los descriptores utilizados fueron "prescripción excesiva", "psicofármacos" " "niño". Los criterios de inclusión fueron artículos de revistas, ensayos clínicos, ensayos clínicos, aleatorizados o no aleatorizados, estudios de casos y controles, estudio de cohortes, acceso abierto, publicados en inglés, portugués, español, entre 2010 y 2021 y con edades comprendidas entre 2 y 18 años. Resultados: Los psicofármacos presentados son ansiolíticos, antipsicóticos, antidepresivos, antieplécticos y psicoestimulantes utilizados para controlar conductas externalizantes e internalizantes. Los agentes externalizantes, a su vez, son prevalentes en los niños, presentando divergencia de prescripciones y síntomas según las edades y el sexo abordado. Consideraciones finales: Así, la prescripción de psicofármacos es inadecuada e indiscriminada en la mayoría de los casos, no respetando las edades mínimas de prescripción, diagnóstico e indicación de cada fármaco y clase.

Palabras clave: Prescripción inadecuada, Psicofármacos, Infancia.

\section{INTRODUÇÃO}

Os psicofármacos, são substâncias químicas, naturais ou sintéticas e são utilizadas para alterar o funcionamento mental dos usuários. Os sedativos, ansiolíticos, antipsicóticos, antidepressivos e psicoestimulantes são prescritos para o plano terapêutico para determinados diagnósticos, tais como: depressão, transtorno bipolar e hiperatividade (OLIVEIRA JRF, et al., 2021; CINTRA KC, et al., 2019).

Uma patologia psiquiátrica é caracterizada por transtornos significativos que afetam o cognitivo, o emocional e/ou o comportamental, refletindo, assim, uma disfunção. Dentre estes transtornos mentais podem ser citados, as crises de pânico, a ansiedade, a depressão, o suicídio, a esquizofrenia, a breve reação de psicose e os distúrbios de alimentação, tais como anorexia nervosa e a bulimia. A quinta edição do Manual Diagnóstico e Estatístico de Transtornos Mentais (DSM-5), fornece a equipe clínica critérios diagnósticos de diversos transtornos mentais, mas sua utilização no diagnóstico infantil é ainda limitada, já que o conhecimento e estudos que abrangem esse grupo ainda é pequeno (MADDEN C, et al., 2014).

É na infância que ocorre o desenvolvimento da criança como indivíduo, tornando -os cada vez mais agentes de seus próprios desejos e necessidades, com subjetividades próprias. O olhar direcionado ao paciente pediátrico precisa ser específico e adaptável para entender tudo o que o cerca e o influencia. A trajetória e o ambiente familiar são de total importância para a avaliação médica antes de qualquer diagnóstico e do uso de psicofármacos (MINISTÉRIO DA SAÚDE, 2013).

Para uma ampla variedade de sintomas psicopatológicos e transtornos psiquiátricos que possuem seu início na infância o diagnóstico torna-se fidedigno quando realizado por meio de uma equipe multidisciplinar composta por médico-pediatra, psiquiatra infantil, psicopedagogo e psicólogo para melhor entender a dimensão e gravidade do diagnóstico e do plano terapêutico projetado. O diagnóstico psiquiátrico se 
apresenta difícil uma vez que não há exames confirmatórios, como os exames de imagem (MADDEN C, et al., 2014).

Em âmbito mundial, os transtornos mentais entre crianças variam de $13 \%$ a $20 \%$ a sua prevalência, o que leva essa faixa etária a prioridade quando se trata de saúde mental. O Brasil, possui taxas de prevalência estimadas em $12,7 \%$ a $19,9 \%$. Na maioria dos casos, o profissional conta apenas com a anamnese e a história clínica do paciente, sendo assim, os envolvidos na prescrição e no acompanhamento clínico de tratamentos com medicamentos psiquiátricos devem estar atualizados em relação à pesquisa sobre outras indicações, a identificação e o tratamento de ef eitos adversos relacionados aos mesmos (CEBALLOS GY, et al., 2019).

No entanto, não há acesso aos serviços especializados e apenas $20 \%$ das crianças no Brasil recebe atendimento necessário. O diagnóstico psiquiátrico infantil é ainda mais delicado, uma vez que o profissional para chegar ao diagnóstico e fazer a devida prescrição deve saber diferenciar entre comportamentos fisiológicos e psicopatológico infantil. Além disso, deve saber ouvir, atentar-se ao psicopatológico, sem preconceitos e julgamentos, levando a criança a um ambiente confiável e totalmente favorável aos seus s inais e sintomas (MADDEN C, et al., 2014; CEBALLOS GY, et al., 2019).

Segundo a Organização Mundial da Saúde (OMS), metade de todas as prescrições dos medicamentos acontece incorretamente. Em geral, os psicofármacos são prescritos para os pacientes pediátricos sem justificativas plausíveis ou sem acompanhamento. São indicados com frequência por profissionais não especializados em prescrições de psicofármacos, psiquiatria e experiência em psicopatologia, como pediatras, médicos de família e comunidade e até mesmo médicos neurologistas. Sendo assim, uso inadequado desses medicamentos em pacientes infantis tem acontecido de forma frequente, assim como ocasionais abusos (DAMASCENO MR, et al., 2019; BIEDERMAN J, et al., 1990)

O tratamento medicamentoso nos transtornos psiquiátricos costuma ser fundamental para a abordagem de um tratamento bem-sucedidos, que também pode incluir outros tipos de intervenções, como a psicoterapia ou as terapias comportamentais. Quando esses agentes são usados de forma correta e são prescritos adequadamente podem aliviar e tratar sinais e sintomas (BIEDERMAN J, et al., 1990).

De tal maneira, esta revisão teve como objetivo analisar o padrão das prescrições dos psicofármacos em crianças, relacionando com a idade e gênero. Avaliando, assim, a existência de prescrições inadequadas.

\section{MÉTODOS}

A abordagem metodológica deste trabalho se propõe a um compilado de pesquisa bibliográfica de abordagem qualitativa e caráter descritivo por meio de uma revisão integrativa da literatura. As bases de dados utilizadas foram o National Library of Medicine (PubMed), Biblioteca Virtual em Saúde (BVS) e Directory of Open Access Journals (DOAJ).

A busca pelos artigos foi realizada por meio dos descritores: "over prescribing", "psychotropic drugs" e "child" utilizando o operador booleano "and". Os descritores citados foram usados apenas na língua inglesa e são encontrados nos Descritores de Ciências da Saúde (DeCS).

Foi realizada essa revisão de literatura, conforme o estabelecimento do tema, definição dos parâmetros de elegibilidade, definição dos critérios de inclusão e exclusão. Após isso, houve a pesquisa das publicações nas bases de dados e das informações encontradas em cada uma delas e, porfim, a exposição dos resultados para a discussão do tema.

Seguindo essa sistemática, após a pesquisa dos descritores nos sites, foram estabelecidos critérios de inclusão e exclusão. Ocorreu a utilização de filtros de pesquisa como journal article e clinical trial. Também foram usados os seguintes filtros: artigos de livre acesso, artigos publicados em inglês, português, espanhol, faixa etária, sendo incluso criança pré-escolar de 2-5 anos, criança: 6-12 anos e Adolescente: 13-18 anos. Foram incluídos todos os artigos originais, ensaios clínicos, randomizados ou não randomizados, estudos de caso-controle e estudos de coorte. 
Além disso, foi critério de inclusão o recorte temporal de publicação de 2010 a 2021, devido á escassa fonte de artigo científicos envolvendo a temática específica de idade e prescrições. Os critérios de exclusão são artigos de revisão de literatura, resumos e metanálise. Todos os artigos que constaram em duplicação ao serem selecionados pelos critérios de inclusão, foram excluídos. Os demais artigos excluídos não estavam dentro do contexto abordado, fugindo do objetivo da temática sobre a prescrição de psicofármacos para crianças.

\section{RESULTADOS}

Após a associação de todos os descritores nas bases pesquisadas foram encontrados 321 artigos. Foram encontrados 201 artigos na base de dados PubMed, 118 artigos na Biblioteca Virtual em Saúde e dois artigos na base de dados DOAJ. Após a aplicação dos critérios de inclusão e exclusão foram selecionados 17 artigos na base de dados PubMed, um artigo no DOAJ e 16 artigos na BVS, sendo que 11 artigos foram retirados por estarem duplicados entre as plataformas PubMed e BVS e um artigo foi retirado por estar em d uplicado nas plataformas DOAJ e BVS, resultando em quatro artigos dos selecionados na BVS, totalizando para análise completa 22 artigos, conforme apresentado na Figura 1.

Figura 1 - Fluxograma de identificação e seleção dos artigos selecionados nas bases de dados PubMed, Biblioteca Virtual em Saúde e Doaj.

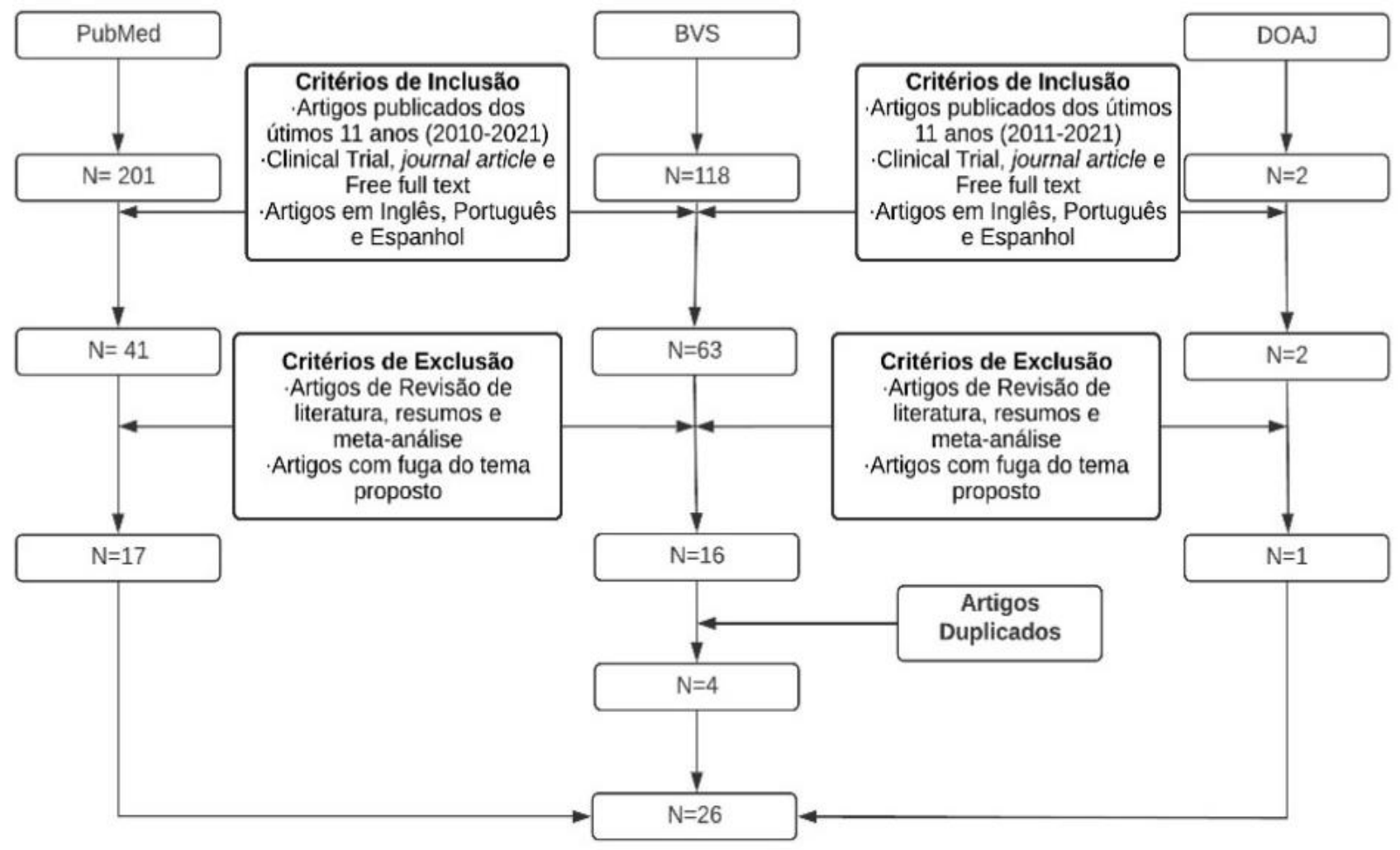

Fonte: Oliveira CRV, et al., 2021.

Os 22 artigos selecionados, os psicofarmácos relatados são ansiolíticos, antipsicóticos, antidepressivos, antiepléticos e psicoestimulantes. São eles: Fluoxetina, Sertralina, Escitalopram, Citalopram, Imipramina, Metilfenidato, Anfetamina, Diazepam, Alprazolam, Zolpidem, Zopiclone, Ciammazina, Risperidona, Olanzapina, Clozapina, Carbamazepina, lamotrigina, Quetiapina e Ácido valpróico. Foram avaliados os resultados dos trabalhos selecionados e construido um quadro comparativo, na qual é composta pelo número de indivíduos abordados nos estudos, ano de publicação, principais prescrições, principal sexo a receber tais prescrições e faixa etária conforme apresentado no Quadro 1. 
Quadro 1 - Caracterização dos artigos conforme ano de publicação, número de indivíduos abordados e principais conclusões das prescrições em relação ao sexo e faixa etária abordada.

\begin{tabular}{|c|c|c|c|c|}
\hline Autor (Ano) & $\mathbf{N}$ & Prescrição & Sexo & Idade \\
\hline $\begin{array}{l}\text { Cuddy E e Currie } \\
J(2020)\end{array}$ & 2.201 .566 & Medicamentos de bandeira vermelha. & Feminino & 10 a 18 anos \\
\hline $\begin{array}{c}\text { Jack RH, et al. } \\
(2020)\end{array}$ & 4.349 .638 & $\begin{array}{l}\text { Antidepressivos: fluoxetina e } \\
\text { sertralina. }\end{array}$ & Feminino & 5 a 17 anos \\
\hline $\begin{array}{l}\text { Newlove-Delgado } \\
\text { T, et al. (2019). }\end{array}$ & 9390 & $\begin{array}{l}\text { Psicotrópicos: antidepressivos e } \\
\text { ansiolíticos. }\end{array}$ & Feminino & 10 a 20 anos \\
\hline $\begin{array}{l}\text { Sultan RS, et al. } \\
(2019)\end{array}$ & 187.563 & $\begin{array}{l}\text { Antipsicóticos + psicoestimulante } \\
\text { (metilfenidato, anfetamina) }\end{array}$ & Masculino & 3 a 24 anos \\
\hline $\begin{array}{l}\text { Sidorchuk A, et al. } \\
\text { (2018) }\end{array}$ & 3.726 .818 & Benzodiazepínico: diazepam & Feminino & 0 a 24 anos \\
\hline $\begin{array}{c}\text { Bonnot O, et al. } \\
(2017)\end{array}$ & 941.857 & $\begin{array}{l}\text { Antipsicóticos: ciammazina e } \\
\text { risperidona. }\end{array}$ & Masculino & 0 a 17 anos. \\
\hline $\begin{array}{l}\text { Tsai C, et al. } \\
\text { (2017) }\end{array}$ & 70.055 & $\begin{array}{c}\text { Risperidona, alprazolam, fluoxetina e } \\
\text { zolpidem. }\end{array}$ & Feminino & 12 a 17 anos \\
\hline $\begin{array}{c}\text { Chon MW, et al. } \\
(2017)\end{array}$ & 2190 & Escitalopram e fluoxetina. & Masculino & 6 a 18 anos \\
\hline $\begin{array}{l}\text { Nielsen ES, et al. } \\
(2017)\end{array}$ & 29.851 & Fluoxetina e imipramina & Feminino & $\leq 17$ anos \\
\hline $\begin{array}{c}\text { Olashore } A \text {, et al. } \\
(2017)\end{array}$ & 238 & $\begin{array}{c}\text { Antipsicóticos atípicos: risperidona, } \\
\text { olanzapina e quetiapina. }\end{array}$ & Masculino & $\leq 17$ anos \\
\hline $\begin{array}{c}\text { Abbas S, et al. } \\
(2016)\end{array}$ & 4.29000000 & Benzodiazepínico: diazepam & Feminino & 0 a 2 anos. \\
\hline $\begin{array}{l}\text { Cook BL, et al. } \\
(2016)\end{array}$ & 22.524 & $\begin{array}{l}\text { Antidepressivo, antipsicótico e } \\
\text { psicoestimulante }\end{array}$ & Masculino & 5 a 17 anos \\
\hline $\begin{array}{l}\text { Hart I, et al. } \\
(2016)\end{array}$ & 1.100000 & $\begin{array}{c}\text { Drogas hipnóticas/sedativas, } \\
\text { psicoestimulantes e antidepressivos. }\end{array}$ & Masculino & $<18$ anos \\
\hline $\begin{array}{l}\text { John A, et al. } \\
(2016)\end{array}$ & 358383 & Antidepressivo: citalopram & Feminino & 6 e 18 anos \\
\hline $\begin{array}{l}\text { Olfson M, et al. } \\
(2016)\end{array}$ & 3.147 .352 & Os antipsicóticos e psicoestimulantes & Masculino & 3 a 24 anos \\
\hline $\begin{array}{c}\text { John AL, et al. } \\
\text { (2015) }\end{array}$ & 311.343 & $\begin{array}{l}\text { Ansiolítico: diazepam. } \\
\text { Hipnótico: zopiclone. }\end{array}$ & Feminino. & 6 a 18 anos \\
\hline Akram G (2015) & 234 & Antipsicótico: risperidona & Masculino & 4 a 14 anos \\
\hline $\begin{array}{l}\text { O'Sullivan K, et al. } \\
\text { (2015) }\end{array}$ & $\begin{array}{l}188.833> \\
<311.579\end{array}$ & Benzodiazepínico: diazepam & Masculino & 0 a 11 anos \\
\hline $\begin{array}{l}\text { Bachmann CJ, et } \\
\text { al. (2014) }\end{array}$ & 431.386 & Antipsicótico atípico: risperidona & Masculino & 0 a 19 anos \\
\hline $\begin{array}{l}\text { Murray ML, et al. } \\
\qquad(2014)\end{array}$ & 5.651 & $\begin{array}{c}\text { Psicotrópicos: Metilfenidato, } \\
\text { risperidona, diazepam e ácido } \\
\text { valpróico. }\end{array}$ & Feminino & 0 a 24 anos \\
\hline $\begin{array}{l}\text { Saloner B, et al. } \\
\text { (2014) }\end{array}$ & 419.226 & $\begin{array}{c}\text { Antipsicóticos de segunda geração } \\
\text { (Clozapina, risperidona, quetiapina e } \\
\text { olanzapina) }\end{array}$ & - & 3 a 18 anos \\
\hline $\begin{array}{l}\text { Hsia Y, et al. } \\
\text { (2010) }\end{array}$ & 641.670 & $\begin{array}{l}\text { Antiepléticos: ácido valpróico, } \\
\text { carbamazepina e lamotrigina. }\end{array}$ & - & 0 a 18 anos \\
\hline
\end{tabular}

Fonte: Oliveira CRV, et al., 2021.

Dos vinte e dois artigos selecionados, dez (45,4\%) avaliaram o sexo feminino na prescrição de psicofarmacos. Dessas prescrições, seis constam antidepressivos, sendo eles: Fluoxetina, Sertralina, Imipramina e Citalopram. Dentre esses, foram mais prescritas a Fluoxetina e a faixa etária dominante foram maiores de 3 anos. Já os ansiolíticos estão presentes em sete das prescrições, sendo eles: Diazepam e Alprazolam. O mais prescrito foi o Diazepam que 62,5\% dessas prescrições tem seu início antes do primeiro 
ano de idade. Nas prescrições do sexo feminino, apenas uma das prescrições possuem psicoestimulantes e duas possuem antipsicóticos.

O sexo masculino, foi avaliado também por dez dos artigos $(45,4 \%)$, que por sua vez, possui sete prescrições de antipsicóticos sendo eles: Risperidona, Ciammazina, Olanzapina e Quetiapina. O principal foi a Risperidona e $54,5 \%$ iniciam apenas para crianças maiores que três anos. Além disso, de todas as prescrições, apenas quatro possuem psicoestimulantes sendo eles: Metilfenidato e Anf etamina. O mais usado é o Metilfenidato e $60 \%$ das prescrições dos psicoestimulantes, possuem seu público-alvo maiores de três anos. Nas prescrições do sexo masculino, apenas três prescrições possuem antidepressivos e duas prescrições apresentam ansiolíticos que raramente são usados em monoterapia.

Apenas dois dos 22 artigos não abordam o sexo como fator influenciador para a prescrição dos antipsicóticos e a classe de antipléticos que foram o ácido valpróico, carbamazepina e lamotrigina. Oito artigos não delimitaram as idades na infância avaliando como menores de 18 anos. Todas as prescrições dos psicofármacos independente do sexo e faixa etária da prescrição são usadas até 18 anos de idade ou mais, sem variação significativa.

\section{DISCUSSÃo}

O mecanismo de ação de um psicofármado é caracterizado pela interação química que uma determinada substância ativa exerce com o receptor produzindo uma resposta terapêutica usada para o tratamento de doenças psíquicas. Dentre as classes utilizadas, os antipsicóticos são divididos em derivados fenotiazídicos, derivados tioxantênicos, butirofenonas e difenilbutilaminas e agentes psicóticos diversos. Possui como ação a depressão seletiva do sistema nervoso central (JACK RH, et al., 2020; NEWLOVE-DELGADO T, et al., 2019).

No tratamento da ansiedade, os ansiolíticos e os antidepressivos são drogas de primeira escolha. Os ansiolíticos são classificados em carbamatos de propanodiol e compostos relacionados, benzodiazepinas e compostos diversos, já os antidepressivos, por sua vez, como o nome sugere, são utilizados também no tratamento da depressão. Tal classe é dividida em compostos tricíclicos, inibidores da MAO, sais de lítio, carbonato de estrôncio e os antidepressivos diversos. Tal medicação possui como mecanismo de ação as vias monoaminérgicas, na qual há influência na produção, no armazenamento e na recaptação das monoaminas (DAMASCENO MR, et al., 2019; CUDDY E e CURRIE J, 2020)

Dos 22 artigos selecionados, oito não delimitaram as idades já que não houve subdivisão significativa na infância. Em um presente estudo, Heloisa HA (2000) relata que em crianças e adolescentes as prescrições de antidepressivos se resumem, especificamente, a Fluoxetina o que corrobora com os resultados do quadro 1 (HELOISA HA, 2000). Os Inibidores Seletivos de Recaptação de Serotonina (ISRS) quando utilizados na infância, apresentam cefaléia, náusea, anorexia, dor abdominal, tontura, sonolência, insônia, nervosismo, ansiedade, inquietação motora, sudoração excessiva e urticária. Tais reações variam de organismo para organismo e a presença deles não a impossibilita de tolerar e responder bem a outro antidepressivo, apenas alterando sua classe com observação de 15 a 20 dias para uma resposta terapêutica adequada (SULTAN RS, et al., 2019; BONNOT O, et al., 2017; CHON MW, et al., 2017; COOK BL, et al., 2016).

A ANVISA, entre 2009 e 2011, relata que o consumo do metilfenidato aumentou $75 \%$ entre crianças e adolescentes na faixa dos 6 aos 16 anos e tem sido escolha no tratamento do controverso do Transtorno de Déficit de Atenção e Hiperatividade (TDAH) (SILVA CM, et al., 2018; SIDORCHUK A, et al., 2018; HART I, et al., 2016).

De acordo com Dias PF, et al. (2020), no mercado brasileiro, o único psicoestimulante disponível é a Ritalina e é considerado um psicofármaco seguro e eficaz em crianças acima dos seis anos de idade. Já em casos de uso em crianças menores de quatro anos, seus ef eitos colaterais são bem maiores e a sua eficácia é reduzida (DIAS PF, et al., 2020). Os tratamentos medicamentosos no TDAH só entram como escolha terapêutica quando há quadros de narcolepsia, quando a reorganização do padrão de vida e de higiene do sono já foram utilizados e não são suficientes para o paciente o que leva a divergência com o quadro 1 que 
há prescrições baseadas na sintomatologia (HELOISA HA, 2000; TSAI C, et al., 2017; NIELSEN ES, et al., 2017; OLASHORE A, et al., 2017).

Os delírios e alucinações, sintomas que caracterizam manifestações psicóticas, são tratados e utilizados como primeira escolha os antipsicóticos, como demonstra Heloisa HA et al (2000). O uso dos antipsicóticos atípicos em crianças, ainda não existem trabalhos que comprovem as doses específica (HELOISA HA, et al, 2000; AKRAM, G., 2015). Quadros como a Esquizofrenia na infância e os Transtornos Invasivos do Desenvolvimento (TID), só respondem ao uso de atípicos e nessas condições se faz necessário como droga de primeira escolha em clientela pediátrica a risperidona ou a olanzapina para a substituição ao anteriormente prescrito haloperidol (JOHN A, et al. ,2016; OLFSON M, et al., 2016;).

Abbas S, et al. (2016), demonstra que o diazepam tem uma porcentagem de prescrições para crianças de 0 a 2 anos. Os benzodiazepínicos são utilizados como primeira escolha em adultos por possuírem boa resposta para o tratamento de ansiedade e insônia. Já na infância, os distúrbios de sono e as manifestações de ansiedade são frequentemente resolvidas com intervenções no ambiente do que com drogas psicoativas. A prescrição dessa classe não deve possuir um plano terapêutico a longo prazo, já que possui grandes ef eitos viciantes e torna-se necessárias doses maiores em longo empo de uso. As alterações cognitivas e sedação podem ocorrer e ocasionam prejuízos no aprendizado escolar, além das reações paradoxal, irritabilidade, crises de raiva, hiperatividade ou agressividade dose dependente de benzodiazepínicos (ABBAS $S$, et al, 2016; HELOISA HA, 2000; JOHN AL, et al., 2015; BACHMANN CJ, et al., 2014).

Outro ponto que merece destaque, é a distribuição do tratamento psicoterápicos em homens e mulheres, sendo os dois vulneráveis, mas encaminhados aos tratamentos psicofármacos distintos pelas queixas diferenciadas. As queixas como dificuldade de concentração, atenção e aprendizagem são mais frequentes no sexo masculino desde a infância, uma vez que, o sexo feminino, caracterizado muitas vezes pela submissão, obediência e passividade apresentam sintomas relacionado a neuróticos graves, tais sintomas são mais toleráveis e dificilmente serão vistos como problemáticos nesse gênero (SALONER B, et al., 2014; MURRAY ML, et al., 2014).

Em vista disso, Dias PF, et al. (2020) relata conforme o sexo masculino e feminino, verificou-se uma maior quantidade de meninos com dificuldade de aprendizado quando comparado as meninas. Assim, crianças que necessitam de apoio escolar, sem especificar, 40\% relataram o uso de psicotrópicos. Logo, a medicalização psicoestimulante é maior de meninos, em relação ao sexo feminino. Somando a esses dados, também evidenciou o predomínio do sexo masculino, em crianças de 5 a 7 anos, para o uso de psicotrópicos. Além disso, a relação entre comportamentos externalizantes como a hiperativid ade, desatenção e comportamentos internalizantes como a ansiedade, depressão e retraimento, sendo os externalizantes mais propensos a encaminhamentos ao serviço de saúde e mais prevalentes em meninos mostrando assim a divergência de prescrições abordada (DIAS PF, et al., 2020; HSIA Y, et al., 2010; O'SULLIVAN K, et al., 2015).

\section{CONSIDERAÇÕES FINAIS}

Os psicofármacos são inevitáveis nos transtornos psiquiátricos infantis e levam o indivíduo ao controle da doença. Dessa forma, foi observada uma inadequada e indiscriminada prescrição de psicotrópicos, na maioria dos casos, não respeitando as idades mínimas para prescrição, o diagnóstico e as indicação de cada medicamento e classe. A conscientização dos profissionais responsáveis pelo diagnóstico e a capacitação necessária para traçar tratamento seja ele ou não farmacológicos. Odiagnóstico e prescrição devem ser feitos pelo médico psiquiatra associado a equipe multidisciplinar de forma individualizado e bem atento a toda trajetória. Além disso, informações simples sobre o tratamento e seu uso podem melhorar a qualidade de vida da criança e a adesão.

\section{REFERÊNCIAS}

1. ABBAS S, et al. Psychopharmacological Prescriptions in Children and Adolescents in Germany. Dtsch Arztebl Int., 2016;113: 396-403.

REAMed | Vol. 2 | DOI: https://doi.org/10.25248/REAM ed.e9382.2022 
2. AKRAM G. Profiling psychotropic discharge medication from a children's psychiatric ward. Int J Clin Pharm, 2015;37: 753-757.

3. BACHMANN CJ, et al. Antipsychotic prescription in children and adolescents: an analysis of data from a German statutory health insurance company from 2005 to 2012. Dtsch Arztebl Int., 2014;111: 25-34.

4. BIEDERMAN J, STEINGARD R. Psicofarmacologiaem niños y adolescentes: un manual para el clinico. Washington, DC: OPS, 1990.

5. BONNOT O, et al. Influência do status socioeconômico nas prescrições de antipsicóticos entre jovens na França. BMC Psychiatry, 2017;17: 82.

6. CEBALLOS GY, et al. Perfil de utilização do serviço do Centro de Atenção Psicossocial da Criança e do Adolescente no Brasil:2008 a 2012. Braz J Psychiatry, 2019; 41: 138-147

7. CHON MW, et al. Prescription Pattern of Antidepressants for Children and Adolescents in Korea Based on Nationwide Data. J Korean Med Sci, 2017; 32: 1694-1701.

8. CINTRA KC, et al. Abordagens farmacológicas em psicofármacos. Rev Inic Cient Ext., 2019.

9. COOK BL, et al. Examining psychotropic medication use among youth in the U.S. by race/ethnicity and psychological impairment. General hospital psychiatry, 2017; 45: 32-39.

10. CUDDY E, CURRIE J. Treatment of mental illness in American adolescents varies widely within and across areas. Proceedings of the National Academy of Sciences of the United States of America, 2020;117(39):24039-24046.

11. DIAS PF, et al. Contexto e consequências do uso de psicofármacos em crianças e adolescentes. Revista Educação em Saúde, 2020; 8:1

12. HARTZ I, et al. Psychotropic drug use among 0-17 year olds during 2004-2014: a nationwide prescription database study. BMC Psychiatry, 2016;16: 12.

13. HELOISA HA. Princípios gerais do emprego de psicofármacos. Rev. Bras. Psiquiatr, 2000;22: 40-41.

14. HSIA Y, et al. TEDDY Network of Excellence. Comparison of antiepileptic drug prescribing in children in three European countries. Epilepsia, 2010;51: 789-96.

15. JACK RH, et al. Incidence and prevalence of primary care antidepressant prescribing in children and young people in England, 1998-2017: A population-based cohort study. PLoS medicine, 2020;17:7.

16. JOHN A, et al. Recent trends in primary-care antidepressant prescribing to children and young people: an e-cohort study. Psychol Med, 2016;46: 3315-3327.

17. JOHN AL, et al. Recent trends in the incidence of anxiety and prescription of anxiolytics and hypnotics in children and young people: An e-cohort study, Journal of Affective Disorders, 2015;183: 134-141.

18. MADDEN C, et al. Treating our youngest patients: psychotropic medications in early childhood. Mo Med, 2014; 111 : 207-211.

19. MINISTÉRIO DA SAÚDE. Secretaria de Atenção à Saúde. Departamento de Atenção Básica. Cadernos de Atenção Básica: Saúde Mental. $2013 . \quad$ Disponível em: https://bvsms.saude.gov.br/bvs/publicacoes/cadernos_atencao_basica_34_saude_mental.pdf. Acessado 06 de jan de 2022.

20. MURRAY ML, et al. Pharmacological treatments prescribed to people with autism spectrum disorder (ASD) in primary health care. Psychopharmacology (Berl), 2014;231:1011-1021.

21. NEWLOVE-DELGADO T, et al. Prescribing for young people with attention deficit hyperactivity disorder in UK primary care: analysis of data from the Clinical Practice Research Datalink. ADHD Atten Def Hyp Disord, 2019; 11: $255-262$.

22. NIELSEN ES, et al. Trends in Off-Label Prescribing of Sedatives, Hypnotics and Antidepressants between Children and Adolescents - A Danish, Estudo Nationwide Register - Based. Basic Clin Pharmacol Toxicol, 20171; (20): 360367.

23. OLASHORE A, et al. Prescribing pattern of psychotropic medications in child psychiatric practice in a mental referral hospital in Botswana. Pan Afr Med J., 2017;26: 83.

24. OLFSON M, et al. Stimulant Treatment of Young People in the United States. J Child Adolesc Psychopharmacol, 2016;26: 520-526.

25. OLIVEIRA JRF, et al. Descrição do consumo de psicofármacos na atenção primária à saúde de Ribeirão Preto, São Paulo, Brasil. Cadernos de Saúde Pública, 2021.

26. O'SULLIVAN K, et al. Benzodiazepine prescribing in children under 15 years of age receiving free medical care on the General Medical Services scheme in Ireland. BMJ Open, 2015;5.

27. SALONER B, et al. Second-generation antipsychotic use among stimulant-using children, by organization of medicaid mental health. Psychiatr Serv., 2014; 65.

28. SIDORCHUK A, et al. Benzodiazepine prescribing for children, adolescents, and young adults from 2006 through 2013: A total population register-linkage study. PLoS Med, 2018;15.

29. SILVA CM, et al. Medicalização da queixa escolar e o uso de psicofármacos como resposta à questões comportamentais. Estudos e Pesquisas em Psicologia, 2018; 18: 738-754.

30. SULTAN RS, et al. Antipsychotic Treatment Among Youths With Attention-Deficit/Hyperactivity. JAMA Netw Open., 2019;2(8):e1911650..

31. TSAI CY, et al. Psychotropic medications prescribing trends in adolescents: A nationwide po pulation-based study in Taiwan, International Journal for Quality in Health Care, 2017;29: 861-866. 